\title{
Design Process of an Area-Efficient Photobioreactor
}

\author{
Jan-Willem F. Zijffers • Marcel Janssen • \\ Johannes Tramper • René H. Wijffels
}

Received: 6 September 2007 / Accepted: 16 December 2007 /Published online: 12 February 2008

(C) The Author(s) 2008

\begin{abstract}
This article describes the design process of the Green Solar Collector (GSC), an area-efficient photobioreactor for the outdoor cultivation of microalgae. The overall goal has been to design a system in which all incident sunlight on the area covered by the reactor is delivered to the algae at such intensities that the light energy can be efficiently used for biomass formation. A statement of goals is formulated and constraints are specified to which the GSC needs to comply. Specifications are generated for a prototype which form and function achieve the stated goals and satisfy the specified constraints. This results in a design in which sunlight is captured into vertical plastic light guides. Sunlight reflects internally in the guide and eventually scatters out of the light guide into flat-panel photobioreactor compartments. Sunlight is focused on top of the light guides by dual-axis positioning of linear Fresnel lenses. The shape and material of the light guide is such that light is maintained in the guides when surrounded by air. The bottom part of a light guide is sandblasted to obtain a more uniform distribution of light inside the bioreactor compartment and is triangular shaped to ensure the efflux of all light out of the guide. Dimensions of the guide are such that light enters the flat-panel photobioreactor compartment at intensities that can be efficiently used by the biomass present. The integration of light capturing, transportation, distribution and usage is such that high biomass productivities per area can be achieved.
\end{abstract}

Keywords Photobioreactor - Sunlight - Efficiency · Light guide $\cdot$ Design

J.-W. F. Zijffers $(\bowtie) \cdot$ M. Janssen $\cdot$ J. Tramper $\cdot$ R. H. Wijffels Food and Bioprocess Engineering Group, Wageningen University, 6700 EV Wageningen, the Netherlands

e-mail: jan-willem.zijffers@wur.nl

\section{Introduction}

Ideally, a photobioreactor for production of biomass should catch all sunlight available at the allocated spot, and transport, channel, and distribute it in such a way into the cultivation vessel that all caught light energy is used for biomass formation. Biomass productivities per area in reported photobioreactors are limited by suboptimal circumstances in the reactor, limiting biological efficiency, or by a suboptimal design limiting light supply into the reactor. High yields can only be achieved by linking photobioreactor design to the biological processes inside. The efficiency of the photobioreactor is determined by the integration of: light capturing, light transportation, light distribution, and light usage.

This article describes the design process of the Green Solar Collector (GSC), a light- and area-efficient photobioreactor for the cultivation of microalgae on sunlight. Light-efficient production of algal biomass has been reported in experiments using single flat-panel photobioreactors (Qiang et al. 1998a, b). These reactors have a large light capturing reactor surface per reactor volume and therefore optimal illumination of the biomass can be achieved. However, area-efficient production is difficult to achieve using flat-panel reactors. The tilt angle, the direction the panel faces, i.e. facing east-west or northsouth, and the number of panels per area all influence the total productivity per unit ground surface. Shading always influences irradiance levels on the surface of flat-panel reactors that are close together (Pulz et al. 1995), which is required if use of all sunlight per ground area is desired. Creating flat-panel reactor compartments through internal illumination by light channeling and distributing elements (light guides) in a larger vessel (Fig. 1), as presented by Janssen et al. (2003), can solve the problems in the delivery of sunlight and the use of all light on the reactor area. 
Fig. 1 Design of a rectangular air-lift photobioreactor with light redistributing plates and external light collection (Janssen et al. 2003)

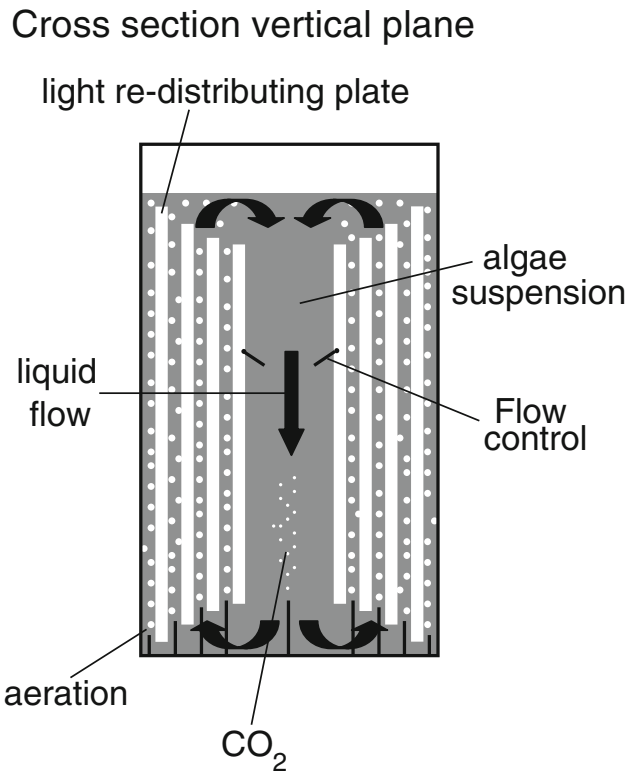

Cross section horizontal plane

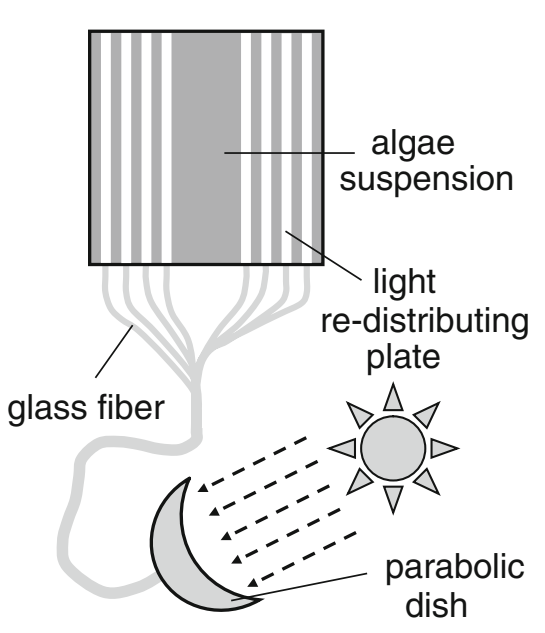

To combine a complete coverage of the ground surface and high productivity, sunlight is captured by lenses on top of the GSC (Fig. 2) and is directed to the photobioreactor compartment through light guides. These light guides are flat rectangular sheets of plastic, guiding the light downward into the algal suspension. The light guides distribute the high-intensity direct solar irradiance over a larger area inside the reactor to lower the sunlight intensity without losing light energy. The combination of decreased sunlight intensities, a short light path between the light guides inside the liquid phase of the photobioreactor, resembling a flatpanel photo-bioreactor, and intensive mixing of the liquid phase by rising air bubbles create an environment to achieve high photosynthetic yields. The goal of the GSC is to efficiently use light energy incident on the bioreactor surface for the production of algal biomass.

The following design goals were set for the Green Solar Collector:

1. All sunlight, falling on top of the reactor must be concentrated into light guides.

2. All captured light must be fully channeled through the light guides toward the bioreactor compartment.

3. All channeled light must be homogeneously scattered from the light guides into the bioreactor compartment.

4. All scattered light must be efficiently used for the production of biomass.

To enlarge the economical feasibility of using the reactor the following additional constraints were set:

1. It must be easy to install, for instance on rooftops of industrial complexes where ample waste $\mathrm{CO}_{2}$ is available.

2. It must be made of cheap and durable materials.
3. It must accommodate an easy to control and robust process.

These goals and constraints have yielded a prototype in which the four composing system elements - light capturing, light channeling, light scattering, and biomass productionare integrated (Fig. 2). The prototype is placed on top of one of our university buildings and the efficiency of light use will be evaluated by cultivation of microalgae.

The article has been split up in the design of all GSC elements through separately addressing each design goal in the order as stated. In the end, all are integrated and the design of the complete system is discussed.

\section{Capturing of Sunlight}

All light energy falling on the top surface of the Green Solar Collector must be captured into light guides. Sunlight

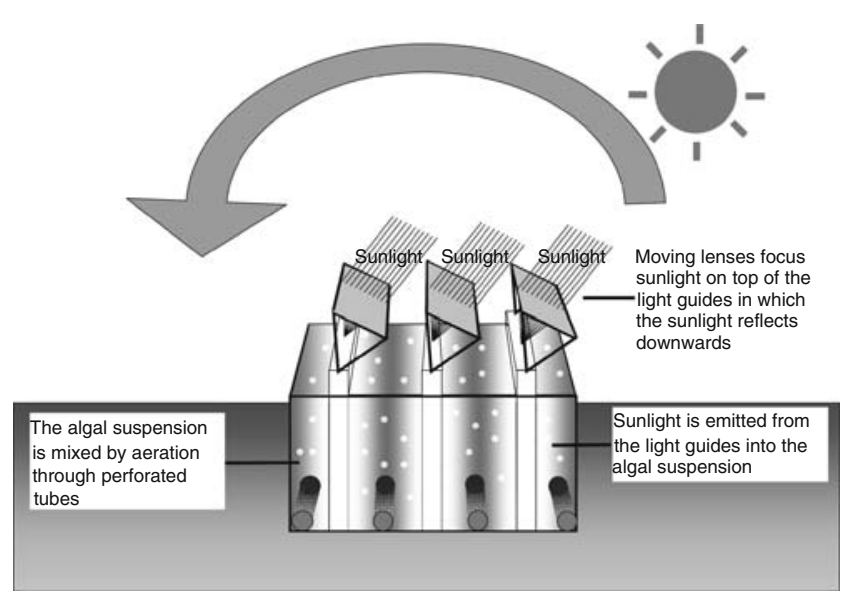

Fig. 2 The Green Solar Collector 


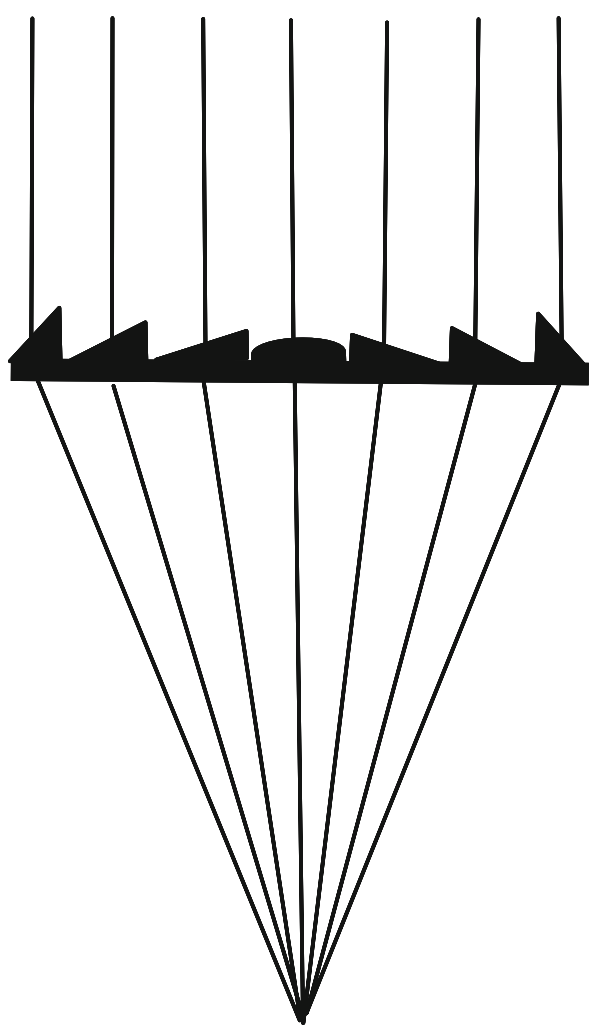

Fig. 3 Focussing of light by a Fresnel lens

must be focused in a line on top of the flat, rectangular plastic light guides to have a good distribution of light energy over the light guide surface. Linear or cylindrical lenses can be used to focus sunlight into a straight line on top of these guides. Light falling on the cylindrical curved surface of convex cylindrical lenses refracts toward the center of the lens, forming a line at a certain distance from the lens. On linear Fresnel lenses the curved surface is replaced by parallel prisms on a flat surface; because of the difference in angle of the prisms, light is refracted toward the focal line (Fig. 3). Fresnel lenses have the same energy concentrating effect as conventional convex linear or cylindrical lenses but are less expensive and less heavy.

Parallel placement of linear Fresnel lenses enables the focusing of almost all sunlight falling on the reactor surface into a number of parallel lines. Rotation over an east-west and north-south axis, called dual-axis rotation, of a lens or a mirror as in the "Himawari" solar light collection device (Mori 1985) and the solar fiberoptic minidish concentrators (Feuermann et al. 2002; Gordon 2002) enables tracking of the sun. Correct dual-axis positioning of linear Fresnel lenses enables full focusing of sunlight on rectangular light guides during the entire year and an almost full capturing of sunlight as stated in design goal 1 .

Positioning the lenses A linear Fresnel lens focuses light into a line. Light does not need to enter perpendicularly from all directions to be focused into a line (Fig. 4). Light only needs to enter perpendicularly with respect to the width of the lens (Fig. 4, top view). A linear Fresnel lens focuses light within a 2-dimensional plane; parallel rays incident over the entire length and perpendicular to width of the lens are focused into a line. The focal distance is fixed and depends on the lens (Fig. 3). In case the light hits the lens surface nonperpendicularly in the length direction, the line of focus is closer to the lens (Fig. 4). The light refracts out of the lens with the same angle as it enters in the length direction, whereas the focal length, the length of the lines leaving the lens in Fig. 3 is fixed. Focusing light in a line, even when light enters nonperpendicular, makes the lens ideal for focusing of light into the light guide. However, the distance between the lens and light guide needs to be adjustable to keep the line of focus on the light guide.

Two control strategies can be applied for positioning the lens. The sun can be tracked using a light sensor mounted on the lens to position the lens perpendicular to the sun or the position of the lens can be programmed. Both need precise dual-axis positioning of the lens. Tracking the sun using a sensor would require repositioning of the lenses when the sun is intermittently blocked by clouds. Programming the position of the lens is more efficient because the position of the sun on the horizon can be precisely calculated (Duffie and Beckman 1974), resulting in a correct position of the lens during the entire day.

Rotation of the system holding the lens over the sheet (Fig. 5, rotation over axis "X") positions the width of the lens perpendicular to the sun. The distance between the lens and sheet is decreased by rotating the four legs holding the lens "A" over axis "Y" (Fig. 6). The rotation can take place independent of the rotation over axis " $\mathrm{X}$ ". The rotation over axis " $Y$ " is called the angle of hinge of the lens; the rotation over axis " $\mathrm{X}$ " is called the angle of rotation of the lens. The distance between lens and sheet is determined by the
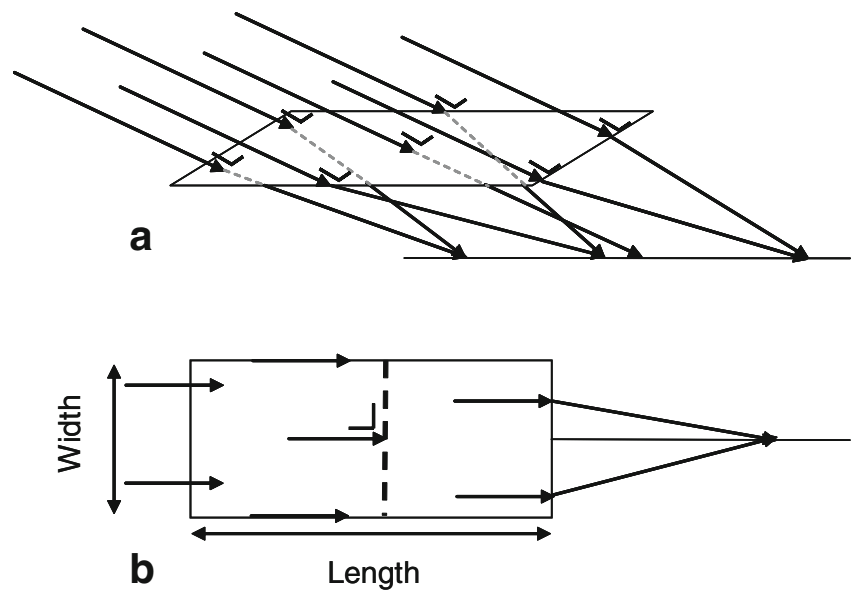

Fig. 4 Focusing of light into a line by a linear lens. a 3-dimensional side view; b 2-dimensional view from the top 


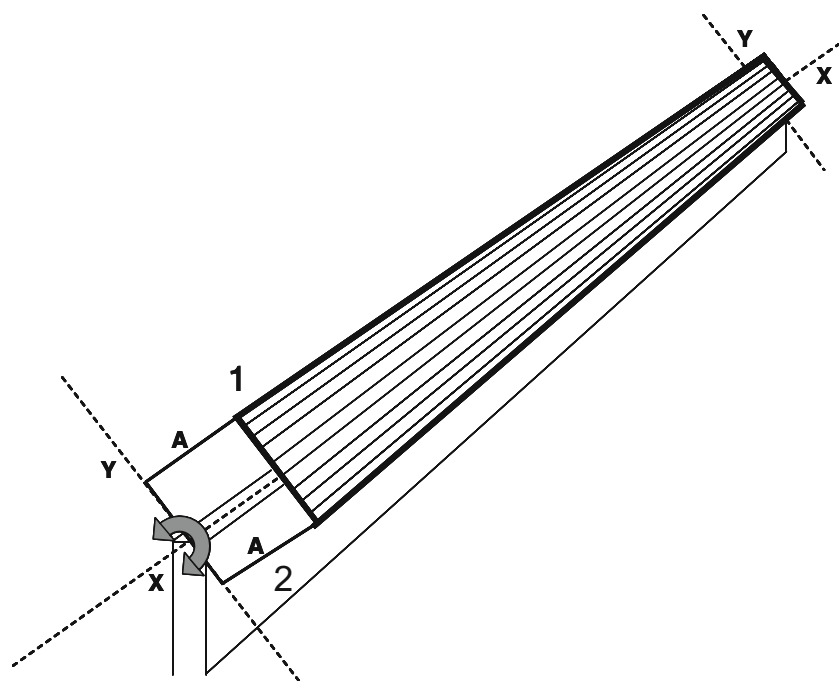

Fig. 5 Rotation of the system holding the lens (1) over axis " $\mathrm{X}$ ", being the top of the light guide (2). The system consists of legs " $A$ " and axis "Y"

incoming angle of the sun; the diagonal parallel to the sun rays between the middle of the lens and top of the light guide in the 2-dimensional plane of focus has to equal the focal length "F" of the lens. In other words, the legs "A," positioning the lens (Figs. 5 and 6), need to point toward the sun in the sky to have a correct positioning of the lens.

Calculating the correct position of the lenses The position of the sun on the horizon during the year can be calculated based on geographical location and date and time (Duffie
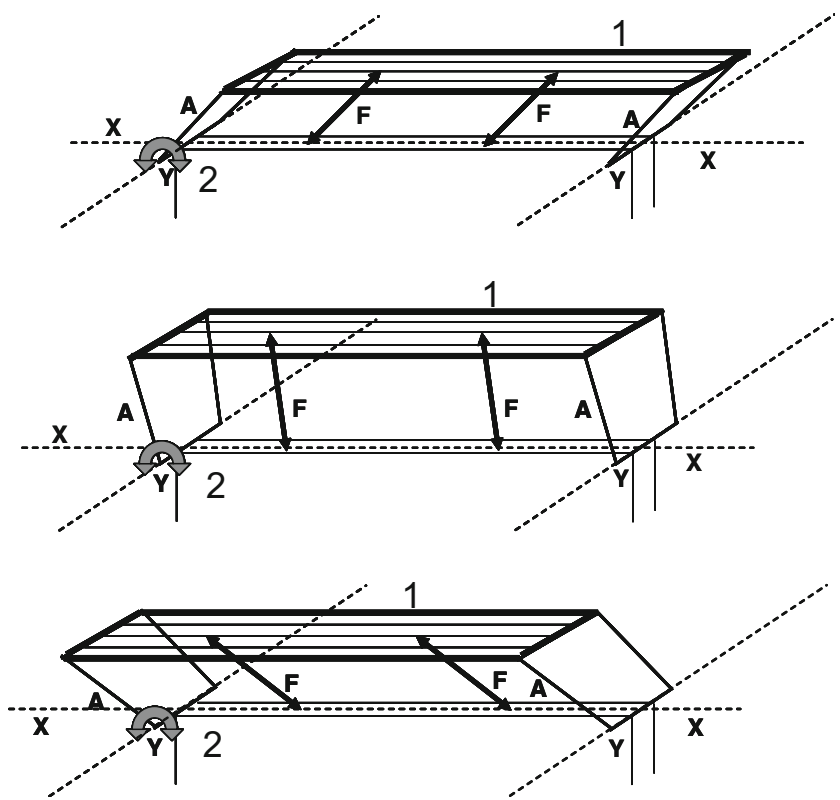

Fig. 6 Different angles of hinge of the lens. Legs "A" of the system holding the lens (1), rotate over axis "Y" to decrease the distance between lens (1) and light guide (2). The diagonal between the middle of the lens and light guide, equals the focal distance " $F$ "

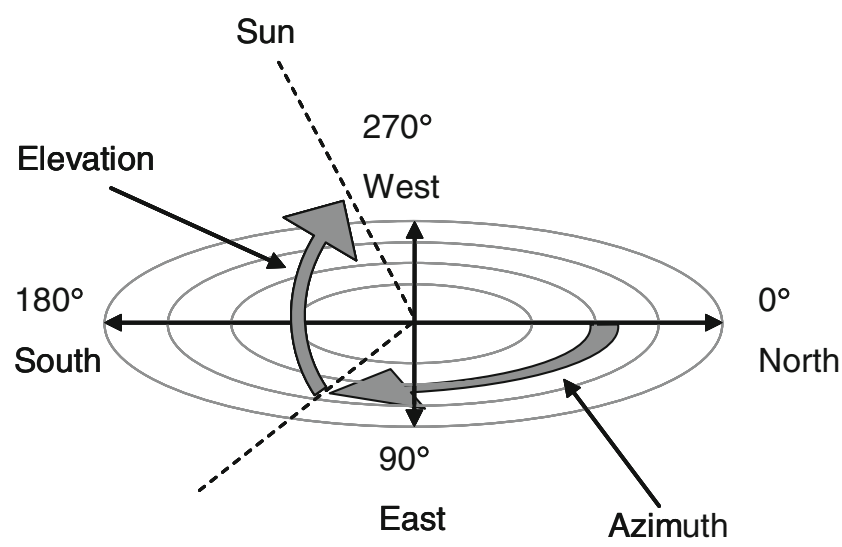

Fig. 7 Azimuth and elevation angle of the sun

and Beckman 1974). The position of the sun is expressed by two angles. The azimuth angle, the position of the sun on the horizon, e.g., commonly referred to as amount of degrees north, east, south, or west (Fig. 7), and the angle of elevation, the angle of the altitude of the sun in the sky relative to a horizontal surface. The position of the lens is calculated based on these angles. The angle of rotation and the angle of hinge are calculated based on the elevation angle and the azimuth angle relative to the orientation of the reactor as described in more detail in Appendix 1.

The angle of rotation and hinge during a number of days in Wageningen, the Netherlands, during the year is illustrated in Figs. 8 and 9. As an example the Green Solar Collector (GSC) is positioned in a north-south orientation, meaning the light guides stretch from north to south. However, the GSC can be placed in any orientation and maintain a good focussing of sunlight on the light guide.

A $90^{\circ}$ angle of rotation means that the lens is in a horizontal position, a $90^{\circ}$ angle of hinge means the legs "A" positioning the lens are upright. At a $0^{\circ}$ and $180^{\circ}$ rotation angle the lens is in a vertical position. At a $0^{\circ}$ and $180^{\circ}$ angle of hinge the lens is positioned downward on top of the light guide.

The position of the lens precisely follows the trajectory of the sun (Figs. 8 and 9). The sun rises in the east and the lens has to be rotated to a vertical position; facing east. Therefore angle of rotation at sunrise is $180^{\circ}$ during the entire year. The angle of hinge at sunrise is determined by the azimuth angle (Fig. 7) at sunrise. During summer the rises in the northeast, which means the angle of hinge is larger than $90^{\circ}$ to point the legs holding the lens to the north. The combination of the angle of hinge and rotation points the legs toward the northeast. During the 21st of March the sun rises in the east, therefore, the angle of hinge is $90^{\circ}$ and the legs holding the lens are upright pointing to the east.

During every day, the lens rotates to a horizontal position at solar noon, when the sun is located in the south. The angle of hinge at this time equals the elevation angle of 
the sun and again the legs holding the lens are pointing to the sun. During the afternoon the sun descends towards the west where it sets. The angle of hinge during the afternoon increases to follow the sun when it descends from the south towards the north. The rotation angle decreases to rotate the lens towards the west until it is vertical at sunset.

In a north-south-oriented reactor the angle of hinge is the rotation over the east-west axis of the reactor to position the lens with respect to the north-south position of the sun. The rotation angle is a rotation over the northsouth axis of the reactor to position the lens with respect to the east-west position of the sun. The angle of hinge and rotation can be adjusted to position the lenses in other reactor orientations.

\section{Channeling of Light to the Bioreactor Compartment}

Transport and distribution of light is integrated in the light guide (Fig. 2). Sunlight is transported efficiently by total internal reflection in the light guide. All light entering the guide is channeled to the bottom of the guide, where it is scattered into the algal suspension inside the bioreactor. In the design of the GSC, care had to be taken that light actually enters the light guide and that it reflects inside the light guide when surrounded by air and refracts out of the guide when surrounded by the algal suspension. Whether light reflects from or refracts into the light guide depends on the angle at which the light hits the top of the guide, the refractive index of the material of which the light guide is made, and the shape of the guide.

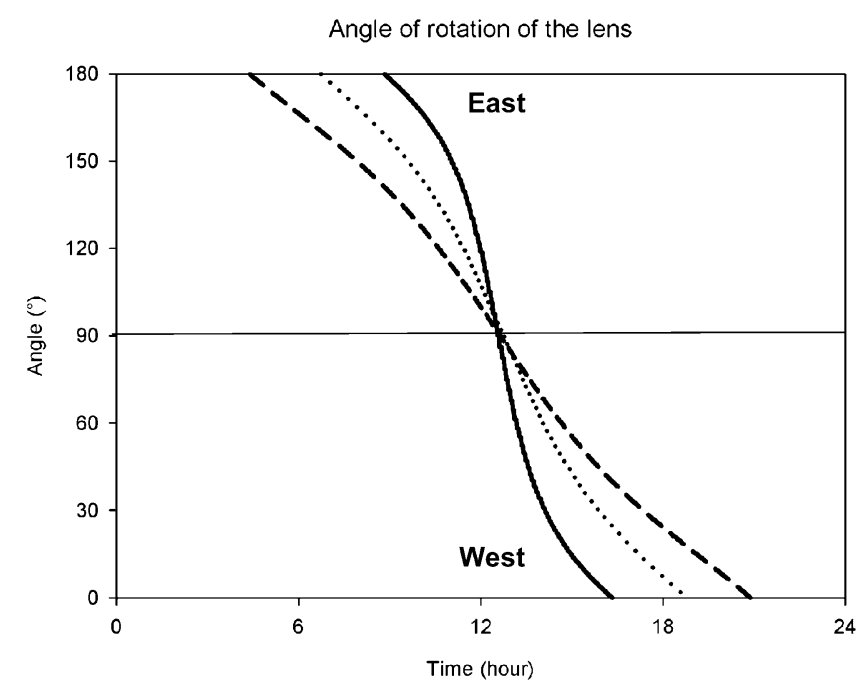

Fig. 8 Angle of rotation in a north-south oriented GSC located in Wageningen, the Netherlands - 21st of December $\cdots \cdots 2$. 21 st of March - - 21st of June
Angle of hinge of the lens

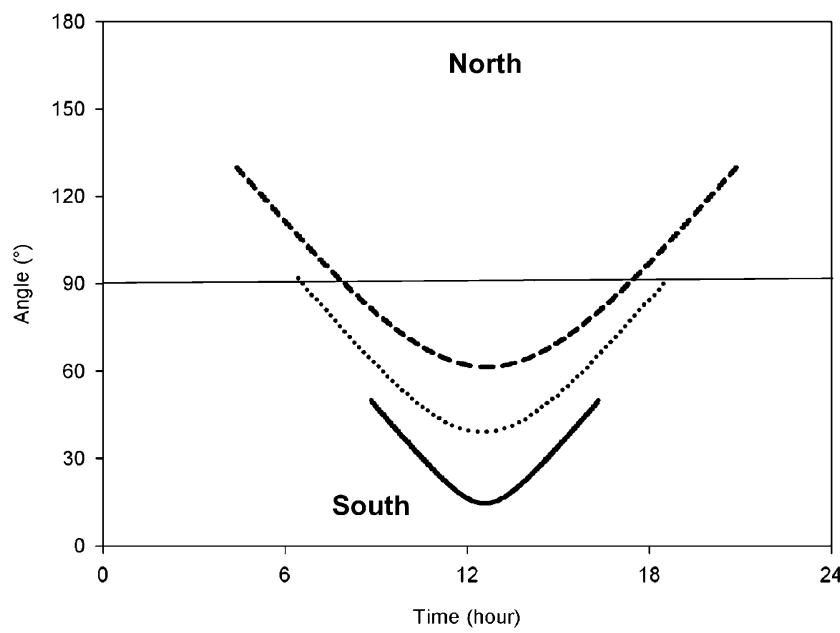

Fig. 9 Angle of hinge in a north-south oriented GSC located in Wageningen, the Netherlands - 21st of December $\cdots \cdots 2$ st of March --21 st of June

Material of the light guide Whether light reflects from or refracts into the horizontal top of the light guide is calculated using Snell's law and Fresnel's formula (Sears 1974) (Appendix 2). Light coming from air always refracts into a clear material with a higher refractive index than air. At small angles, relative to the normal, the highest percentage of light refracts into this clear material, whereas at larger angles the amount of reflection increases but still a certain percentage of light refracts into the material up to an angle of $89^{\circ}$.

Materials with refractive indices close to the refractive index of air cause light to refract out of the guide over its vertical sides, which is undesired on the upper part of the guide where it is not surrounded by the algal suspension (Fig. 10). High refractive indices, on the other hand, cause a large amount of light to reflect at the light guide's point of entry. This also is undesired, because light would not refract into the guide and is not available for the algae. Thus, the refractive index of the guide had to be high enough to ensure total internal reflection when surrounded by air (Ries et al. 1997) but not much higher to limit the reflection on top of the guide.

Whether light reflects internally in the guide or refracts out of the guide at the vertical guide-air interface can again be calculated using Snell's law and Fresnel's formula (Appendix 2). A light guide, surrounded by air and accepting all possible angles on the top surface must have a refractive index higher than 1.415 (Appendix 2). Polymethylmethacrylate (PMMA) a clear transparent plastic with the highest transmittance for visible light compared with other plastics is an ideal material for a light guide. It has the same transmittance as glass and it has a refractive index of 1.49 to 1.50 for visible light, which is larger than the required refractive index of 1.415 . This ensures total internal 


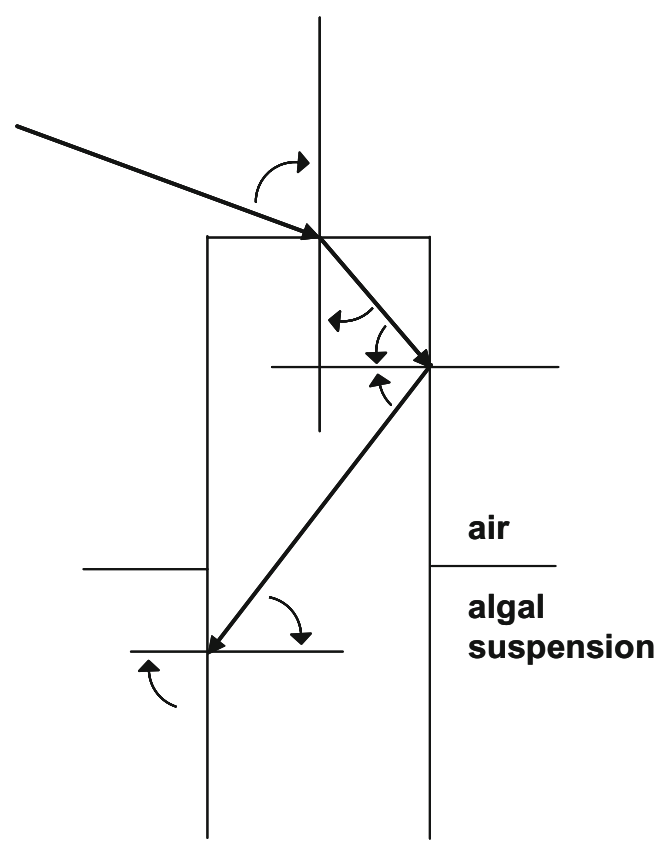

Fig. 10 Light refracts into the light guide. Inside the light guide it reflects internally when surrounded by air and it refracts out of the guide when it is surrounded by the algal suspension

reflection inside the light guide when it is surrounded by air while limiting the reflection of light on top of the guide.

\section{Reflection and Refraction in the Top of the Light Guide}

As calculated in Appendix 2, total internal reflection occurs when light refracts toward the center of the light guide when entering into the top of the guide. Fig. 11 shows that light does not refract towards the center in a guide with a round top surface. Because of the more perpendicular angles of the incoming light on the round top surface, light does not refract sufficiently and total internal reflection does not occur.

More sunlight reflects from the guide's flat top surface than from a round top surface because light enters more perpendicularly on the round surface. However, loss of light energy due to refraction out of the guide because of a round top surface is larger than reflection on top of a flat top surface. Light must refract downward into the guide to be available to the algae. The top surface of the guide is, therefore, flat because it is the best shape to capture and keep light in the guide.

\section{Scattering of Light into the Bioreactor Compartment}

The top part of the light guide is surrounded by air and light needs to reflect internally, but the bottom part is surrounded by the algal suspension and light needs to be scattered from the light guide into the suspension. As calculated in
Appendix 2, the maximum angle for which total internal reflection occurs when surrounded by water is $26.8^{\circ}$ from the vertical sides. Sunlight with an angle between $0^{\circ}$ and $42.2^{\circ}$, from the normal, entering on top of the light guide would still reflect internally and eventually leave through the bottom of a rectangular guide. This is undesired since light has to leave the guide over its left and right surface in order to have a uniform scattering of light out of the light guide surface into the bioreactor compartment.

The only way to ensure that all light leaves the guide over its left and right surface is to change the incident angle of the light on the bottom part of the guide. A triangular shaped bottom part changes the angle at which the light reflects and results in the refraction of all light out of the bottom part of the guide. Fig. 12 shows the refraction and reflection of light on top of and inside the guide. The light guide needs to narrow towards the bottom to let all light refract out of the guide and the tip has to be surrounded by the algal suspension.

The uniformity of scattering of light out of the guide is still under investigation. Preliminary measurements showed that light does not reflect uniformly out of a guide with a smooth surface, and a surface treatment, such as sandblasting, showed a more uniform scattering of light out of the entire surface of the triangular bottom part. Calculations show (data not presented) that all light scatters out of the light guide for the smooth as well as the sandblasted surface.

\section{Efficient Use of Scattered Light}

The use of scattered light by the algae is maximized by creating compartments (Fig. 13, numbered "3") with short light paths and turbulent mixing, similar to the flat-panel compartments described by Qiang et al. (1998a, b). In these
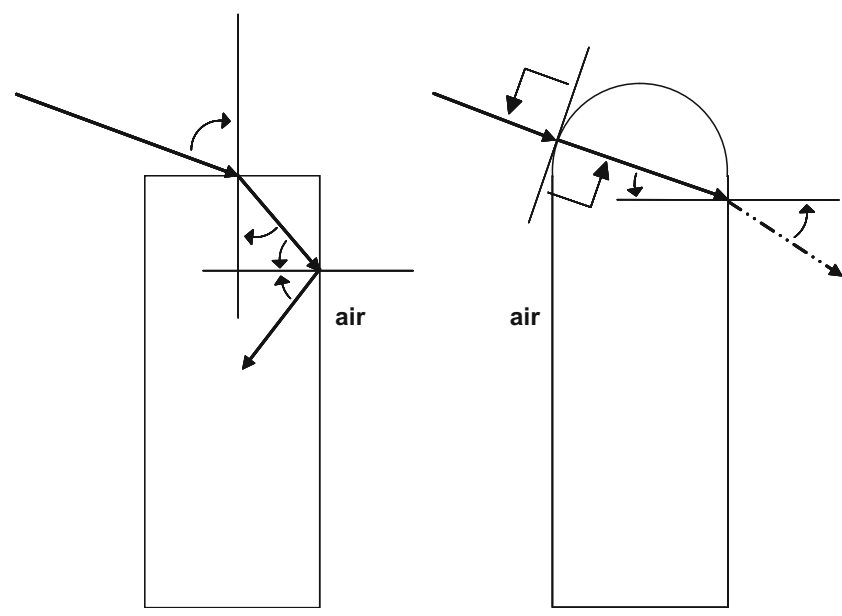

Fig. 11 Influence of the shape of the top of the light guide on the reflection and refraction of light inside the light guide 


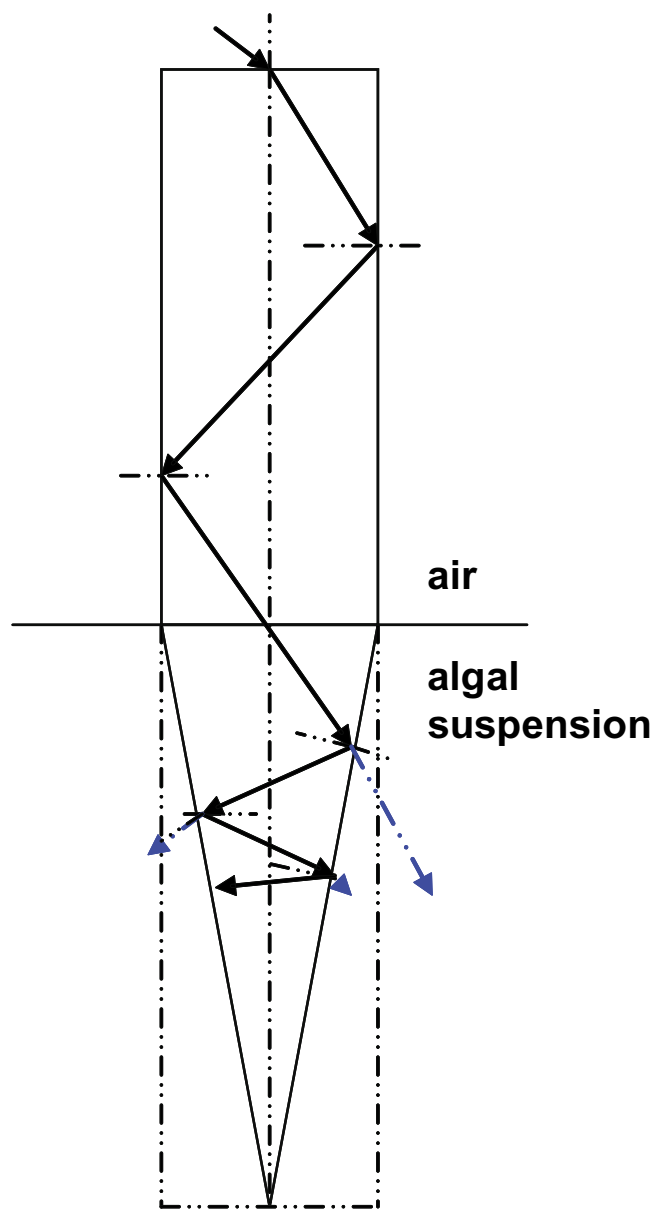

Fig. 12 Reflection of light inside the light guide. Total internal reflection in the top part and total refraction out of the guide in the bottom part

flat-panel photobioreactor experiments up to $20 \%$ of the PAR sunlight energy falling on the reactor surface was stored as biomass; approaching the theoretical maximum conversion efficiency of $21 \%$ of light within the PAR range (Bolton and Hall 1991).

The short light path in a flat-panel photobioreactor, combined with turbulent mixing induced by aeration at the bottom causes a rapid circulation of the algae from the illuminated reactor wall to the dark interior of the reactor. It subjects the algae to intermittent illumination which is suggested to increase the efficiency of use of oversaturating light intensities (Richmond 1996). Well controllable flat-panel compartments with turbulent mixing by aeration also are comprised between the light guide and water jacket in the GSC. Although the width of the compartment in our reactor varies from top to bottom of the compartment, on average it is similar to the width reported by Qiang et al. (1998a, b).

The flat-panel compartment Figure 13 shows the design of the GSC. The light guide separates the reactor into two flat- panel compartments between the left and right water jacket. The light guide resembles the transparent reactor wall of a panel reactor. Instead of being a light transparent barrier between the algal culture and the outside, it now separates two algal culture compartments and delivers light to both compartments. The rectangular light guide, the wall of the water jacket and aeration at the bottom along the length of the reactor, together create an environment similar to a flatpanel photo bioreactor.

\section{Integrating the Four Reactor Elements}

Use of the focused sunlight Light is captured and distributed into the GSC during the entire day, but optimal conditions need to be provided for the algae. The most important is to provide a suitable light intensity in the GSC. In the flat-panel photobioreactor cultivation experiments described by Qiang et al. (1998a, b) the highest volumetric productivity was not achieved in the reactor with the highest efficiency. An efficiency of $20 \%$ was achieved in a panel reactor not fully exposed to sunlight. It was positioned vertically; therefore reflection of light occurred

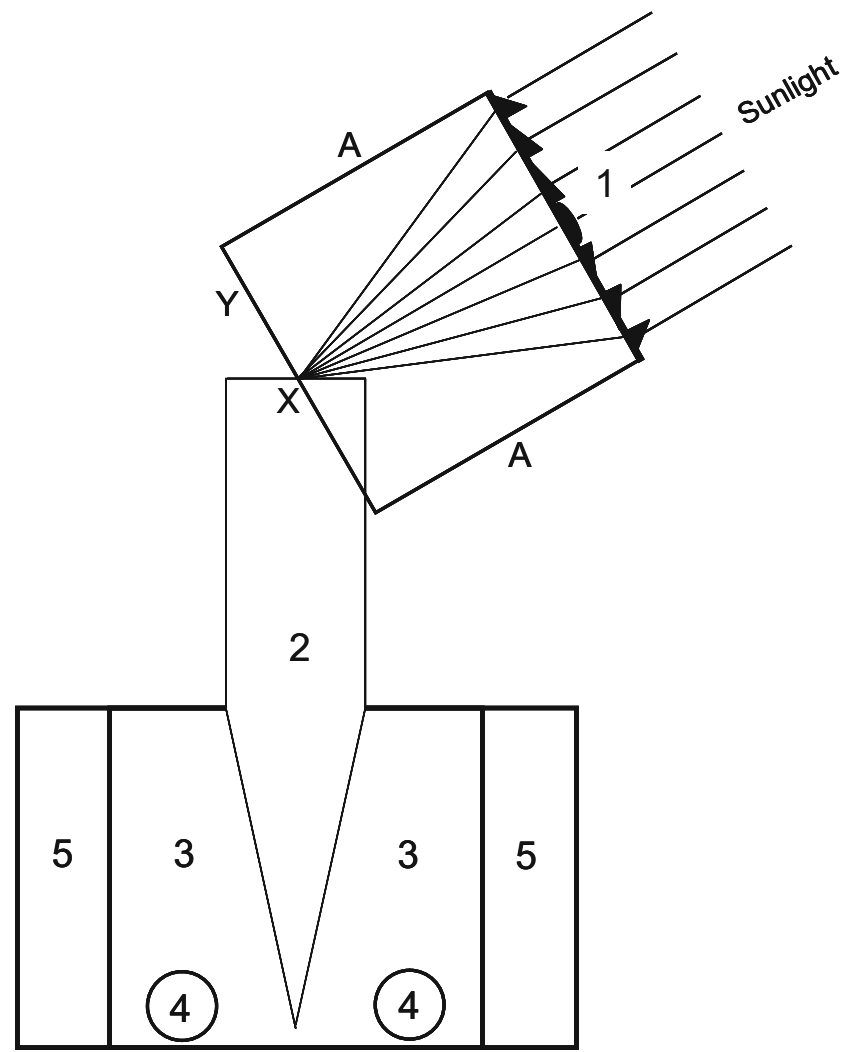

Fig. 13 Cross-section of the photobioreactor. The numbers indicate the following: 1: linear Fresnel lens, 2: light guide, 3: flat-panel reactor compartment, 4 : perforated tube for aeration, 5: water jacket. The letters indicate the following: A: legs holding the lens, $\mathrm{X}$ : axis of rotation of the lens, Y: axis of rotation of the legs "A" 
on the reactor surface, decreasing the light intensity inside the panel reactor.

In the GSC care is taken in supplying sunlight to the algae at the right intensity. If light is captured and transported with $100 \%$ efficiency, only the dimensions of the lens and light guide determine the light intensity in the bioreactor compartment. It then is determined by the ratio of the surface of the lens to the surface of the light guide in the algal culture.

Total design of the bioreactor The GSC contains flat-panel bioreactor compartments of $18-\mathrm{mm}$ (top) to $25-\mathrm{mm}$ (bottom) wide and the maximum light intensity has to be approximately half of the intensity of the sun to have an efficient use of light while having a high biomass productivity. Therefore, the illuminating surface of the light guide has to be the double of the light capturing surface of the lens to dilute the maximum sunlight intensity to the desired intensity. Because the length of the lens and light guide are equal, the height of the triangular shaped bottom part has to be equal to the width of the lens, because light refracts from both sides of the bottom part of the guide.

The GSC is designed to cover large areas by having multiple light guides next to each other in a larger housing. The distance from one light guide to the other is constructed by two flat panel compartments, one water jacket, and the light guide itself. This distance is fixed at $60 \mathrm{~mm}$. To be able to have an unhindered movement of neighboring lenses; a lens with a width of 52 and a focal distance of $51 \mathrm{~mm}$ is chosen. The width of the lens fixes the height of the bottom part of the light guide to $50 \mathrm{~mm}$ as explained above. The width of a flat-panel compartment is constraint by design limitations, but the length of the compartment is not and can stretch up to several meters. Taking into account he rectangular top part of the light guide and the lens, it results in a system that is maximally approximately $200 \mathrm{~mm}$ high and contains 36 liters of algal suspension per square meter.

\section{Discussion}

The real proof of efficient use of light is in determining the actual area biomass yield of the GSC. This will be determined by the integration of: light capturing, light transportation, light distribution, and light usage. In our design, sunlight is captured directly in the light guide to minimize loss of light energy. Previous attempts by others to capture sunlight with lenses and to transport light into a light guide through optical fibers proved less efficient and expensive. Gordon (2002) and Ogbonna et al. (1999) stated that low efficiencies in the delivery of light into the photobioreactor through optical fibers were obtained due to coupling problems between different light guiding fibers and loss of light in transport through the fibers. Costs and construction considerations for large scale optical fiber photobioreactor cultivation systems further limited the application of fibers (Gordon 2002). These considerations and the efficiency problems do not meet the Green Solar Collector's design goals and constraints. Focusing sunlight directly on the light guide is more efficient, because light does not have to be transported over long distances. Light reflects internally without loss in intensity over the small distance where it needs to be transported.

The GSC's light guides increase the illuminated surface per reactor volume and, therefore, resembles the sheets described by Janssen et al. (2003). Multiple light guides next to each other increase the illuminated surface in larger bioreactors. Previously, scratched optical fibers were used to internally illuminate a photobioreactor and to increase the illuminated surface per reactor volume (An and Kim 2000; Matsunaga et al. 1991). Large numbers of optical fibers are needed to have an increased surface to volume ratio compared with noninternal illuminated reactors. The construction and maintenance of large photobioreactor systems containing thousands of optical fibers will not meet the design constraints posed in the introduction; therefore, a sheet of PMMA is used to internally illuminate the photobioreactor compartments in the GSC.

An effect such as shading, common in vertical plate or column reactors placed close together, does not interfere with the light supply. The potential for achieving optimal conditions in our photobioreactor is present due to uniform illumination inside the reactor. Each lens focuses the same amount of sunlight into the light guide, due to the parallel placement of the lenses, creating a similar illumination pattern throughout large areas of the GSC. Process conditions are therefore similar throughout large GSC areas. The lenses shade each other somewhat at low altitudes of the sun, depending on the orientation of the reactor. However, because the lenses are placed parallel, shading is the same on each lens, again leading to the same illumination in all flat-panel compartments.

The GSC has been constructed (Fig. 14) and by visual observation it is clear that the lenses are able to focus sunlight on top of the light guide and that light refracts out of the guide into the algal suspension. The actual efficiency of light supply is still unknown but can be estimated based on the reflection of light on the lens and light guide as shown earlier. Also the uniform illumination over both sides of the bottom part of the light guide is still being investigated.

\section{Conclusions}

The GSC complies with the stated goals and constraints. The lenses capture sunlight coming from all angles through 


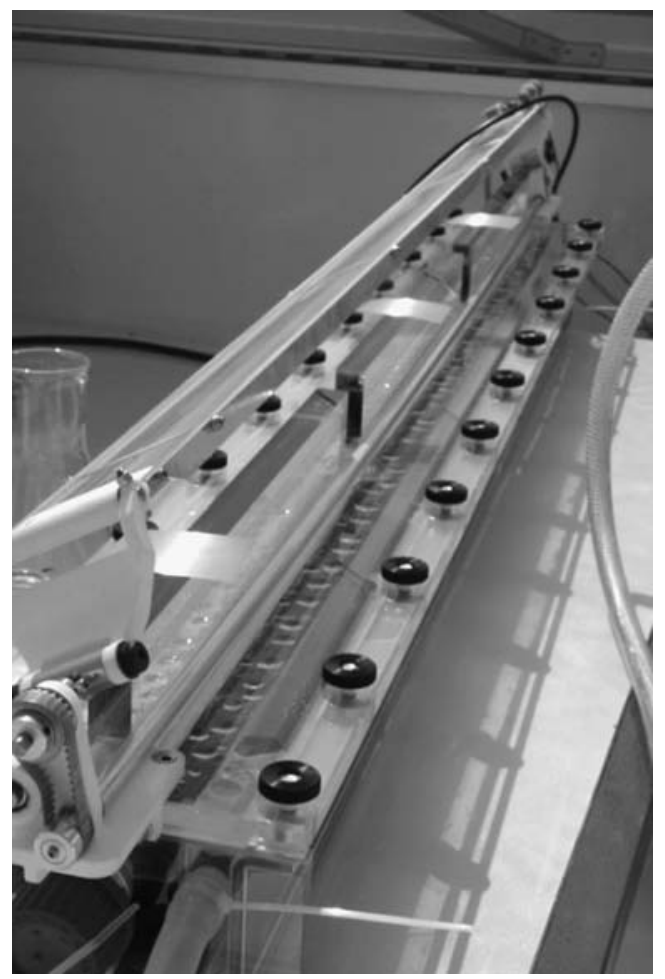

Fig. 14 GSC; the lens in the top of the picture focuses light on top of the light guide (line of focus visible on top of the sheets of paper on the guide). Light refracts internally to be scattered into the algal suspension

dual-axis positioning of linear Fresnel lenses. Sunlight is focused on the light guides in which it propagates by internal reflection and ends up in the algal suspension in the flat-panel compartments.

Almost all light falling on the GSC is intercepted by the lenses during the day, except when the lenses are in a horizontal position. At maximum, $8.7 \%$ of the direct sunlight passes in between the lenses and will strike the transparent reactor cover. The demand to capture all sunlight per ground area in the light guides is therefore almost completely met. Capturing all sunlight into the light guides was not possible, because some space is needed in between lenses to allow unhindered movement of the lenses. This fraction of direct sunlight as well as diffuse sunlight can still enter the reactor compartment through the transparent top reactor cover and is not lost for the microalgae.

The capturing efficiencies of the GSC were calculated based on reflection on the lens and light guide during the day combined with reported direct irradiance values (PVGIS: Geographical Assessment of Solar Energy Resource and Photovoltaic Technology; http://re.jrc.cec.eu.int/pvgis/pv/, January 2007). The capturing efficiency decreases in fall and winter due to the lower elevation angles of the sun (Table 1). The amount of reflection on top of the light guide increases significantly. The different capturing efficiencies presented in Table 1 are related to the chosen orientation of the GSC with respect to the sun (Appendix 1).

The efficiency of scattering from the light guide into the bioreactor compartment is estimated to be $100 \%$ based on the reflection and refraction of light. Therefore, high biomass productivities can be obtained during summer in the Netherlands. During winter the GSC is less efficient due to a lower capturing efficiency. At low latitudes, on the other hand, high productivities can be achieved during the entire year. At these locations the sun has a higher angle of elevation, which reduces the reflection of sunlight on top the light guide. However, clear skies are still a prerequisite for high productivities.

The production costs of the GSC are expected to be higher than those of conventional outdoor photobioreactors, such as horizontal and vertical tubes or vertical panels. The production costs compared with fiber optic photobioreactors (Mori 1985), on the other hand, will be lower because of ease of construction and maintenance and the use of cheap materials (PMMA). The most expensive part will be the system to position the lenses, but on large areas covered with multiple units all lenses have to be in the same position. The lens positioning system therefore can be integrated in one single system controlling multiple reactor units. Consequently, economy of scales can significantly reduce reactor costs.

A robust process can be run in the GSC because suitable light intensities are combined with turbulent mixing and control of $\mathrm{pH}$ and temperature. The GSC is constructed of durable plastics and is light in weight. The reactor can thus be easily placed on horizontal rooftops and can be an alternative to conventional thermal or photovoltaic solar collectors. A new type of solar collector had been created: "the Green Solar Collector." The real proof of functionality of the designed reactor needs to come from cultivation experiments; conclusions can then be made on the overall efficiency of use of light in the Green Solar Collector.

Table 1 Capturing efficiency of direct light in the GSC in Wageningen, the Netherlands

\begin{tabular}{ll}
\hline Day of the year & Light capturing efficiency \\
\hline 21 st of December & $27 \%-32 \%$ \\
21 st of March & $57 \%-61 \%$ \\
21 st of June & $76 \%-67 \%$ \\
21 st of September & $57 \%-61 \%$ \\
\hline
\end{tabular}


Acknowledgements This research was supported by Senternovem Neo (0268-03-04-03-002), Biopartner (807.04.220), BWP II (EET K 03028), and Solar-H (516510). The system to position the lenses was constructed by TNO Industrial Technology, the Netherlands.

Open Access This article is distributed under the terms of the Creative Commons Attribution Noncommercial License which permits any noncommercial use, distribution, and reproduction in any medium, provided the original author(s) and source are credited.

\section{Appendix 1}

The position of the lenses is calculated based on the elevation angle of the sun and the relative orientation of the reactor to the azimuth angle of the sun. Starting point is a north-south orientation of the reactor, which means that the lenses on top of the reactor stretch from north to south. If the orientation changes, if the reactor for instance stretches from east to west, or northwest to southeast, the azimuth angle has to be adjusted. Equations 1 and 2 yield the position of the lens based on the position of the sun $(\gamma$ and $\alpha)$ and the orientation of the reactor $(\beta)$.

$\tan ($ rotation $)=\tan \left(\frac{\sin (\gamma)}{\cos (\gamma)^{*} \cos (\alpha-\beta)}\right)$

$\sin ($ hinge $)=\sin \left(\sqrt{\left((\sin (\gamma))^{2}+\left(\cos (\gamma)^{*} \cos (\alpha-\beta)\right)^{2}\right)}\right)$

$\alpha=$ recalculated azimuth angle of the sun on the horizon (Table 2 and Fig. 7).

$\gamma=$ angle of elevation of the sun in the sky (Fig. 7).

$\beta=$ orientation of the Green Solar Collector (Table 3).

Table 2 shows the values for $\alpha$ to calculate the position of the lenses. The value for $\alpha$ is continuously changing with the azimuth angle of the sun. The value for $\beta$ (Table 3 ) is fixed with the chosen orientation of the reactor.

Table 2 Redefining the azimuth angle into $\alpha$ to calculate the angle of hinge and rotation
Table 3 Factor $\beta$; correcting the orientation of the photobioreactor to calculate the angle of hinge and rotation

\begin{tabular}{lr}
\hline Orientation & $\beta$ \\
\hline North-South & $0^{\circ}$ \\
Northeast-Southwest & $45^{\circ}$ \\
East-West & $90^{\circ}$ \\
Southeast-Northwest & $45^{\circ}$ \\
\hline
\end{tabular}

A rotation angle of $0^{\circ}$ or $180^{\circ}$ means that the lens is in a vertical position on the left or right side of the light guide. An angle of hinge of $0^{\circ}$ or $180^{\circ}$ means that the lens is positioned on top of the light guide on the left or right side. At an angle of $90^{\circ}$, the lens is in an upward and/or horizontal position. Whether $0^{\circ}$ or $180^{\circ}$ is left or right for the angle of hinge or rotation is a matter of definition, because the path of the sun on the horizon is the same in the morning and the afternoon, where the solar noon functions as the mirror point.

The angle of rotation is calculated by using equation 1 . The result is an angle between $0^{\circ}$ and $90^{\circ}$. The angle of rotation on one side of the light guide is defined as this angle; the one on the other side as $180^{\circ}$ minus this angle. The angle of hinge is calculated by using equation 2 . The result is an angle between $0^{\circ}$ and $90^{\circ}$. The angle of hinge in one direction is defined as this angle; the one in the other direction as $180^{\circ}$ minus this angle.

\section{Appendix 2}

The angle of refraction of light propagating from one material into the other is calculated by using Snell's law. The index of refraction (n) of each material and the angle of incidence $(\theta)$ determine what the angle of refraction or reflection is. When light refracts from a material $\left(\mathrm{n}_{1}\right)$ with a low refractive index into one $\left(\mathrm{n}_{2}\right)$ with a high refractive index the sine of the angle of refracted light $\left(\theta_{2}\right)$ is smaller than of the light entering $\left(\theta_{1}\right)$, so light will be refracted toward the normal. When the refractive index of the material from which light propagates $\left(n_{1}\right)$ is higher, the sine of the angle of refracted light $\left(\theta_{2}\right)$ increases, therefore, it is refracted away from the normal and at larger angles of incident light $\left(\theta_{1}\right)$ it will not refract into the material $\left(\mathrm{n}_{2}\right)$ at all. It then reflects on the interface.

$\frac{n_{1}}{n_{2}}=\frac{\sin \theta_{2}}{\sin \theta_{1}} \quad$ Snell's law

\begin{tabular}{lrr}
\hline North & $0^{\circ}$ & $90^{\circ}$ \\
Northeast & $45^{\circ}$ & $45^{\circ}$ \\
East & $90^{\circ}$ & $0^{\circ}$ \\
Southeast & $135^{\circ}$ & $45^{\circ}$ \\
South & $180^{\circ}$ & $90^{\circ}$ \\
Southwest & $225^{\circ}$ & $45^{\circ}$ \\
West & $270^{\circ}$ & $0^{\circ}$ \\
Northwest & $315^{\circ}$ & $45^{\circ}$ \\
& & \\
\hline
\end{tabular}

.

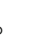 .}

.

政


refracted light determines the amount of reflected light energy. A larger difference causes more reflection. Therefore, the larger the relative difference in refractive index of the materials the higher the amount of reflection.

$R=100 \% *\left[0.5 * \frac{\tan ^{2}\left(\theta_{1}-\theta_{2}\right)}{\tan ^{2}\left(\theta_{1}+\theta_{2}\right)}+0.5 * \frac{\sin ^{2}\left(\theta_{1}-\theta_{2}\right)}{\sin ^{2}\left(\theta_{1}+\theta_{2}\right)}\right]$

Fresnel's formula

$\Theta \quad=$ angle from the normal

$\mathrm{R}$ = percentage of light energy that reflects from the surface

In the case of normal incident irradiance from the air, the reflection can be calculated by using equation 5 .

$R=100 \% *\left(\frac{n_{2}-n_{1}}{n_{1}+n_{2}}\right)^{2}$

When light refracts into a rectangular light guide, it can reflect internally on the vertical sides of the guide, depending on the shape and surrounding material. When light is not refracted toward the normal on the top of the guide, the resulting angle of incidence on the side of the guide is too high to ensure internal reflection.

An example:

Incident light at an angle of $45^{\circ}\left(\theta_{1}\right)$ hits a light guide $\left(\mathrm{n}_{2}=1.8\right)$ from air $\left(\mathrm{n}_{1}=1\right)$. It refracts into the guide at an angle of $23.1^{\circ}\left(\theta_{2}\right)$ and $9.4 \%$ of the light energy is lost due to reflection. The refracted light hits the vertical side of guide at an angle of $90^{\circ}-23.1^{\circ}=66.9^{\circ}\left(\theta_{1}\right)$, relative to the normal. Using Snell's law $\left(\mathrm{n}_{1}=1.8 ; \mathrm{n}_{2}=1\right)$, the angle of refraction on the side of the guide results in an angle larger than $90^{\circ}\left(\theta_{2}\right)$; meaning all light reflects inside the guide.

Incident light at an angle of $45^{\circ}\left(\theta_{1}\right)$ hits a light guide with a different material $\left(\mathrm{n}_{2}=1.2\right)$ from air $\left(\mathrm{n}_{1}=1\right)$. It refracts into the guide at an angle of $36.1^{\circ}\left(\theta_{2}\right)$ and $1.3 \%$ of the light energy is lost due to reflection. The refracted light hits the vertical side of the guide at an angle of $90^{\circ}-36.1^{\circ}=53.9^{\circ}$ $\left(\theta_{1}\right)$, relative to the normal. Using Snell's law $\left(\mathrm{n}_{1}=1.2 ; \mathrm{n}_{2}=\right.$ 1 ), the light refracts out of the guide at an angle of $75.8^{\circ}\left(\theta_{2}\right)$ and only $17.4 \%$ of the light energy is reflected on the interface. The major part of the light energy, $82.6 \%$, is lost as a result of refraction out of the side of the light guide.

Whether light will reflect internally can also be determined by calculating the numerical aperture (NA). It is the sine of the angle of incident light on top of the light guide for which total internal reflection occurs and it determines the applicability of a material as a light guide (Gordon
2002). The angle for which total internal reflection occurs can be calculated using equations 6 and 7 .

$N A=n_{1} * \sin \left(\theta_{\max }\right)$

$N A=\sqrt{\left(n_{2}^{2}-n_{1}^{2}\right)}$

The refractive index only needs to be high enough to ensure total internal reflection in the rectangular light guide when surrounded by air. Taking the maximum angle on the light guide $\left(\theta_{\max }=90^{\circ}\right)$ and the refractive index of air $\left(\mathrm{n}_{1}=\right.$ 1), the NA of the light guide can be calculated by using equation 6 . The refractive index of the light guide $\left(\mathrm{n}_{2}\right)$ needs to be 1.414 to have internal reflection of all captured light as can be calculated from equation 7. At this or higher refractive indices, light at an angle of $90^{\circ}$ refracts into the light guide at an angle of $45^{\circ}$ or lower, while total internal reflection can already occur at angles of $45^{\circ}$ or higher. At lower refractive indices, the angle of refraction will be larger than $45^{\circ}$ and total internal reflection will only occur at angles lower than $45^{\circ}$, resulting in refraction of light out of the sides of the light guide.

The NA also can be calculated, when a PMMA $\left(n_{1}=\right.$ 1.49) light guide is surrounded by water $\left(\mathrm{n}_{2}=1,33\right)$, using equation 7. The maximum acceptance angle on top of the light guide can be calculated by using equation 6 , in which $\mathrm{n}_{1}=1$, because light enters the guide from air. The maximum angle of incident light on top of the light guide for which total internal occurs $\left(\theta_{\max }\right)$ is $42.2^{\circ}$. Incident light with angles smaller than $42.2^{\circ}$ from the normal reflects internally when the light guide is surrounded by water.

\section{References}

An JY, Kim BW (2000) Biological desulfurization in an optical-fiber photobioreactor using an automatic sunlight collection system. J Biotechnol 80:35-44

Bolton JR, Hall DO (1991) The maximum efficiency of photosynthesis. Photochem Photobiol 53:545-548

Duffie JA, Beckman WA (1974) Solar engineering of thermal processes. Wiley, New York

Feuermann D, Gordon JM, Huleihil M (2002) Solar fiber-optic mini-dishconcentrators: first experimental results and field experience. Sol Energy 72:459-472

Gordon JM (2002) Tailoring optical systems to optimized photobioreactors. Int J Hydrog Energy 27:1175-1184

Janssen M, Tramper J, Mur LR, Wijffels RH (2003) Enclosed outdoor photobioreactors: light regime, photosynthetic efficiency, scaleup, and future prospects. Biotechnol Bioeng 81:193-210

Matsunaga T, Takeyama H, Sudo H, Oyama N, Ariura S, Takano H, Hirano M, Burgess JG, Sode K, Nakamura N (1991) Glutamate production from $\mathrm{CO} 2$ by marine Cyanobacterium synechococcus sp. using a novel biosolar reactor employing light-diffusing optical fibers. Appl Biochem Biotechnol 28(9):157-167 
Mori K (1985) Photoautotrophic bioreactor using visible solar rays condensed by Fresnel lenses and transmitted through optical fibers. Biotechnol Bioeng Sympos 15:331-345

Ogbonna JC, Soejima T, Tanaka H (1999) An integrated solar and artificial light system for internal illumination of photobioreactors. J Biotechnol 70:289-297

Pulz O, Gerbsch N, Buchholz R (1995) Light energy supply in plate-type and light diffusing optical-fiber bioreactors. J Appl Phycol 7:145-149

Qiang H, Faiman D, Richmond A (1998a) Optimal tilt angles of enclosed reactors for growing photoautotrophic microorganisms outdoors. J Ferment Bioeng 85:230-236
Qiang H, Zarmi Y, Richmond A (1998b) Combined effects of light intensity, light-path and culture density on output rate of Spirulina platensis (Cyanobacteria). Eur J Phycol 33:165171

Richmond A (1996) Efficient utilization of high irradiance for production of photoautotropic cell mass: a survey. J Appl Phycol 8:381-387

Ries H, Segal A, Karni J (1997) Extracting concentrated guided light. Appl Optics 36:2869-2874

Sears FW (1974) Optics, 3rd edn. Addison-Wesley, Reading, Massachusetts 